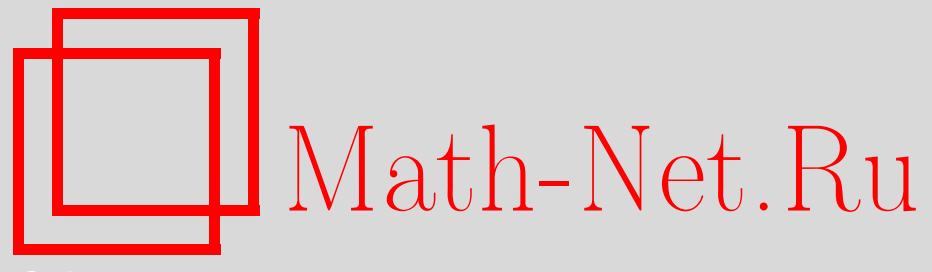

В. А. Ватутин, Редуцированные ветвящиеся процессы в случайной среде: критический случай, Теория вероятн. и ее примен., 2002, том 47, выпуск 1, 21-38

DOI: https://doi.org/10.4213/tvp2959

Использование Общероссийского математического портала MathNet.Ru подразумевает, что вы прочитали и согласны с пользовательским соглашением

http://www . mathnet.ru/rus/agreement

Параметры загрузки:

IP : 54.210 .77 .194

26 апреля 2023 г., 13:54:27

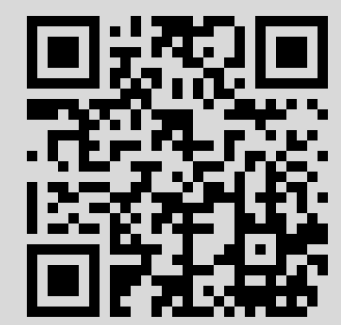




\section{РЕДУЦИРОВАННЫЕ ВЕТВЯЩИЕСЯ ПРОЩЕССЫ В СЛУЧАЙНОЙ СРЕДЕ: КРИТИЧЕСКИЙ СЛУЧАЙ}

Пусть $Z_{n}$ - число частиц в момент $n=0,1,2, \ldots$ в ветвяшемся процессе в случайной среде, $Z_{0}=1$, а $Z_{m, n}$ - число таких частиц в этом процессе в момент $m \in[0, n]$, каждая из которых имеет непустое потомство в момент $n$. Показано, что если производящие функции $f_{k}(s)$ числа потомков частиц $k$-го поколения независимы и одинаково распределены, причем $\mathbf{E} \ln f_{k}^{\prime}(1)=0$, а $\sigma^{2}=\mathbf{E}\left(\ln f_{k}^{\prime}(1)\right)^{2} \in(0, \infty)$, то при некоторых дополнительных условиях последовательность условных процессов

$$
\left\{\frac{1}{\sigma \sqrt{n}} \ln Z_{[n t], n}, t \in[0,1] \mid Z_{n}>0\right\}
$$

сходится по распределению при $n \rightarrow \infty$ к процессу $\left\{\inf _{t \leqslant u \leqslant 1} W^{+}(u)\right.$, $t \in[0,1]\}$ в топологии Скорохода в пространстве $D[0,1]$, где $W^{+}(t)-$ броуновская извилина на $[0,1]$.

Ключевье слова и фразы: критический ветвящийся процесс в случайной среде, редуцированный процесс, функциональная предельная теорема, случайное блуждание.

Ветвящиеся процессы в случайной среде являются довольно популярным объектом исследования [1]-[3], [7], [8], [16], [18]-[26]. Неформальное описание этих процессов в интересующем нас случае выглядит следуюшим образом. Процесс начинается в момент $n=0$ с одной частицы нулевого поколения, которая живет до момента $n=1$ и в конце жизни производит потомство в соответствии с производящей функцией $f_{0}(s)$, причем эта функция является случайной, т.е. выбирается из множества всех вероятностных производяцих функций целочисленных неотрицательных величин в соответствии с некоторым вероятностным распределением. Частицы первого поколения живут до момента $n=2$ и в конце жизни производят потомство в соответствии с общей производящей функцией $f_{1}(s)$, которая является вероятностной копией $f_{0}(s)$, и т.д.

${ }^{*}$ Математический институт им. В. Ф. Стеклова РАН, ул. Губкина, 8, 117966 Москва, Россия; e-mail: vatutin@mi.ras.ru

1) Работа выполнена при финансовой поддержке Российского фонда фундаментальных исследований (коды проектов 99-01-00012, 00-15-96136) и ИНТАС (код проекта 99-01317). 
Пусть $Z_{n}$ - число частиц в процессе в момент времени $n=0,1,2, \ldots$. Закон эволюции $Z_{n}$ может быть задан следующим образом:

$$
Z_{0}=1, \quad \mathbf{E}\left[s^{Z_{n+1}} \mid f_{0}, f_{1}, \ldots, f_{n} ; Z_{0}, Z_{1}, \ldots, Z_{n}\right]=\left(f_{n}(s)\right)^{Z_{n}} .
$$

Ветвящийся процесс в случайной среде называется докритическим, если $\rho=\mathbf{E} \ln f_{0}^{\prime}(1)<0$, критическим, если $\rho=0$, и надкритическим, если $\rho>0$.

В настоящей заметке мы будем исследовать критические ветвящиеся процессы в случайной среде. Известно, что такие процессы вырождаются с вероятностью единица. Нахождение асимптотики вероятности невырождения $\mathbf{P}\left\{Z_{n}>0\right\}$ при $n \rightarrow \infty$ для критических ветвящихся процессов в случайной среде с производящими функциями числа потомков общего вида оказалось сложной задачей. Так, еще в 1976 году М. В. Козлов [7] показал, что если функции $f_{n}(s), n=0,1,2, \ldots$, дробнолинейны, то, при некоторых предположениях о параметрах дробнолинейных функций,

$$
\mathbf{P}\left\{Z_{n}>0\right\} \sim \frac{\beta}{\sqrt{n}}, \quad \beta>0, n \rightarrow \infty,
$$

однако лишь в 2000 году Г. Керстинг и И. Гейгер [26] установили справедливость (2) для произвольных критических ветвящихся процессов в случайной среде.

Положим

$$
\eta_{n}=\frac{f_{n-1}^{\prime \prime}(1)}{2\left(f_{n-1}^{\prime}(1)\right)^{2}}, \quad X_{n}=\ln f_{n-1}^{\prime}(1) .
$$

Теорема 1 ([26]). Если $\rho=0,0<\mathbf{E} X_{1}^{2}<\infty u$

$$
\mathbf{E} \eta_{1}\left(1+\ln ^{+} f_{0}^{\prime}(1)\right)<\infty
$$

то выполнено (2).

Для осуществления следующего естественного шага - доказательства условных предельных теорем о числе частиц в критических процессах в случайной среде при условии невырождения потребовалось также немало времени. Наилучший на данный момент результат принадлежит В. И. Афанасьеву [2]. Для его формулировки нам понадобятся новые обозначения и понятия.

Пусть $W(t), t \in[0, \infty), W(0)=0,-$ стандартное броуновское движение, $W^{+}(t), t \in[0, \infty),-$ броуновская извилина, $\tau=\inf \{t>0$ : $\left.W^{+}(t)=0\right\}$, и

$$
W_{1}^{+}(t)=W^{+}(t \wedge \tau), \quad t \in[0, \infty),
$$

- «остановленная» броуновская извилина. 
Теорема 2. Если $Z_{n}-$ критический ветвящийся прочесс в случайной среде, величинь $\mathbf{E} \eta_{1}, \mathbf{E} X_{1} \eta_{1}, \mathbf{E} X_{1}^{4}, \mathbf{E} \exp \left(-X_{1}\right)$ конечны и, кроме mozo, $\sigma^{2}=\mathbf{E} X_{1}^{2}>0, m o$

$$
\left\{\frac{1}{\sigma \sqrt{n}} \ln \left(Z_{[n t]}+1\right), t \in[0, \infty) \mid Z_{n}>0\right\} \stackrel{D}{\longrightarrow}\left\{W_{1}^{+}(t), t \in[0, \infty)\right\}
$$

где знак $\stackrel{D}{\rightarrow}$ обозначает сходимость по распределению в топологии Скорохода в пространстве $D[0, \infty)$.

3 а м е ч а н и е 1 . Сходимость (4) для $t \in[0,1]$ была впервые доказана в [1] в предположении, что производящие функции числа непосредственных потомков частиц дробно-линейны. В общем случае, но по-прежнему для $t \in[0,1]$, теорема 2 была получена в [8].

Следующим этапом в исследовании ветвящихся процессов является изучение структуры их генеалогических деревьев. Одной из важных характеристик генеалогического дерева является так называемый редуцированный процесс $Z_{m, n}, 0 \leqslant m \leqslant n$, в котором величина $Z_{m, n}$ равна числу частиц в рассматриваемом процессе в момент $m \leqslant n$, имеющих непустое потомство в момент $n$. Свойства редуцированных процессов для ветвящихся процессов Гальтона-Ватсона, марковских ветвящихся процессов с непрерывным временем и процессов Беллмана-Харриса изучались в работах [5], [6], [9]-[15], [22], [23].

Редуцированные ветвящиеся процессы в случайной среде с дробнолинейными производящими функциями числа непосредственных потомков рассматривались в работах [18] и [21]. В настоящей работе мы отказываемся от предположения дробной линейности и доказываем следующее утверждение, которое является основным результатом данной работы.

Теорема 3. Пусть выполнень условия теоремь 2. Тогда

$$
\left\{\frac{1}{\sigma \sqrt{n}} \ln Z_{[n t], n}, t \in[0,1] \mid Z_{n}>0\right\} \stackrel{D}{\longrightarrow}\left\{\inf _{t \leqslant u \leqslant 1} W^{+}(u), t \in[0,1]\right\},
$$

где знак $\stackrel{D}{\rightarrow}$ обозначает сходимость по распределению в топологии Скорохода в пространстве $D[0,1]$.

Заметим, что теорема 3 не может быть выведена из теоремы 2 применением принципа инвариантности Донскера-Прохорова.

Отметим также, что в [18] для случая дробно-линейных производящих функций сходимость (5) была установлена при более слабых моментных предположениях. 
1. Вспомогательные результаты. В дальнейшем нам будет удобно использовать следующие обозначения:

$$
f_{k, n}(s)=f_{k}\left(f_{k+1}\left(\cdots\left(f_{n-1}(s)\right) \cdots\right)\right), \quad 0 \leqslant k \leqslant n-1, \quad f_{n, n}(s)=1 .
$$

Кроме того, мы будем использовать символ $I(A)$ для индикатора события $A$.

Занумеруем частицы $m$-го поколения произвольным, но фиксированным образом. Через $Z_{m, n}^{(k)}, k=1,2, \ldots, Z_{m}, 0 \leqslant m \leqslant n$, будем обозначать размер потомства в момент $n$ в популяции, порожденной $k$-й частицей из числа существовавших в момент $m \leqslant n$. Поскольку мы рассматриваем процессы с дискретным временем, то для упрощения записи всюду, если это требуется по смыслу, величины вида $Z_{n t, n}, f_{n t, n}(0), f_{n t}(0)$ и т.д. будут пониматься при $t \in[0,1]$ как $Z_{[n t], n}, f_{[n t], n}(0), f_{[n t]}(0)$ соответственно.

Для $x>0, N>0, t \in[0,1]$ и $\delta>0$ введем события

$$
\begin{aligned}
& A_{n, t}(x)=\left\{\ln \left(e+Z_{n t, n}\right)>x \sqrt{n}\right\} \\
& B_{n, t}(N)=\left\{\left|\sum_{k=1}^{Z_{n t}}\left(I\left(Z_{n t, n}^{(k)}>0\right)-\left(1-f_{n t, n}(0)\right)\right)\right|\right. \\
&\left.>\sqrt{N Z_{n t}\left(1-f_{n t, n}(0)\right) f_{n t, n}(0)}\right\}, \\
& C_{n, t}(\delta)=\left\{0<Z_{n t}\left(1-f_{n t, n}(0)\right)<e^{n^{1 / 4-\delta}}\right\} .
\end{aligned}
$$

Лемма 1. В условиях теоремь 3 для любых фиксированньх $x>0$, $t \in(0,1)$ и $\delta \in\left(0, \frac{1}{4}\right)$ справедливы соотношения

$$
\begin{aligned}
\limsup _{N \rightarrow \infty} & \limsup _{n \rightarrow \infty} \sqrt{n} \mathbf{P}\left(A_{n, t}(x) B_{n, t}(N)\right)=0, \\
& \limsup _{n \rightarrow \infty} \sqrt{n} \mathbf{P}\left(A_{n, t}(x) C_{n, t}(\delta)\right)=0 .
\end{aligned}
$$

Д о к а з а т е л ь с т в о. Остановим сначала справедливость соотношения (7). В дальнейшем для краткости мы будем писать $\mathbf{P}_{\mathscr{F}}(\cdot)$, $\mathbf{E}_{\mathscr{F}}(\cdot)$ и $\mathbf{D}_{\mathscr{F}}(\cdot)$ для обозначения вероятностей, математических ожиданий и дисперсий, подсчитанных при фиксированной $\sigma$-алгебре $\mathscr{F}$, порожденной случайными величинами $Z_{n t}$ и $f_{n t}(s), f_{n t+1}(s), \ldots, f_{n}(s)$.

Заметим прежде всего, что

$$
\mathbf{E}_{\mathscr{F}} I\left(Z_{n t, n}^{(k)}>0\right)=1-f_{n t, n}(0), \quad \mathbf{D}_{\mathscr{F}} I\left(Z_{n t, n}^{(k)}>0\right)=\left(1-f_{n t, n}(0)\right) f_{n t, n}(0)
$$

Используя эти соотношения и применяя неравенство Чебышева для оценки вероятности под знаком математического ожидания, получаем 
для достаточно больших $n$

$$
\begin{aligned}
\mathbf{P}\left(A_{n, t}(x) B_{n, t}(N)\right) & \leqslant \mathbf{P}\left(Z_{n t}>0, B_{n, t}(N)\right)=\mathbf{E} \mathbf{P}_{\mathscr{F}}\left(Z_{n t}>0, B_{n, t}(N)\right) \\
& \leqslant \mathbf{E}\left[\frac{I\left(Z_{n t}>0\right)}{N Z_{n t}\left(1-f_{n t, n}(0)\right) f_{n t, n}(0)} \mathbf{D}_{\mathscr{F}}\left[\sum_{k=1}^{Z_{n t}} I\left(Z_{n t, n}^{(k)}>0\right)\right]\right] \\
& =N^{-1} \mathbf{P}\left\{Z_{n t}>0\right\} .
\end{aligned}
$$

Эта оценка вместе с (2) влечет (7).

Для доказательства (8) заметим, что

$$
\begin{aligned}
\mathbf{P}\left(A_{n, t}(x) C_{n, t}(\delta)\right) & =\mathbf{E P}_{\mathscr{F}}\left(A_{n, t}(x) C_{n, t}(\delta)\right) \\
& \leqslant \frac{1}{x^{2} n} \mathbf{E}\left[I\left(C_{n, t}(\delta)\right) \mathbf{E}_{\mathscr{F}}\left[\ln ^{2}\left(e+Z_{n t, n}\right)\right]\right]
\end{aligned}
$$

Поскольку

$$
\left(\ln ^{2}(e+x)\right)^{\prime \prime}=\frac{2}{(e+x)^{2}}(1-\ln (e+x))
$$

то функция $\ln ^{2}(e+x)$ вогнута на множестве $x>0$. Этот факт позволяет нам применить неравенство Иенсена для внутреннего математического ожидания в правой части (11) и получить оценку

$$
\mathbf{E}_{\mathscr{F}} \ln ^{2}\left(e+Z_{n t, n}\right) \leqslant \ln ^{2}\left(e+\mathbf{E}_{\mathscr{F}} Z_{n t, n}\right)
$$

Отсюда, используя представление $Z_{n t, n}=\sum_{k=1}^{Z_{n t}} I\left(Z_{n t, n}^{(k)}>0\right)$ и первое из равенств в (9), находим, что при всех достаточно больших $n$

$$
\begin{aligned}
\mathbf{P}\left(A_{n, t}(x) C_{n, t}(\delta)\right) & \leqslant \frac{1}{x^{2} n} \mathbf{E}\left[I\left(C_{n, t}(\delta)\right) \ln ^{2}\left(e+Z_{n t}\left(1-f_{n t, n}(0)\right)\right)\right] \\
& \leqslant \frac{1}{x^{2} n} \ln ^{2}\left(e+e^{n^{1 / 4-\delta}}\right) \leqslant \frac{2}{x^{2} n^{1 / 2+2 \delta}}
\end{aligned}
$$

Легко вицеть, что эти оценки влекут (8).

Лемма доказана.

Следуюшее утверждение имеет, по-видимому, самостоятельный интереc.

Лемма 2. Если $\rho=0,0<\mathbf{E} X_{1}^{2}<\infty u \mathbf{E} \eta_{1}<\infty$, то для любого $v \in(0,1]$ и пюбого $x<0$

$$
\lim _{n \rightarrow \infty} \mathbf{P}\left\{\frac{1}{\sigma \sqrt{n}} \ln \left(1-f_{0, n v}(0)\right)<x\right\}=\mathbf{P}\left\{\inf _{0 \leqslant u \leqslant v} W(u)<x\right\} .
$$

3 а м е ч а н и е 2. Отметим, что аналог леммы 2 для марковских ветвящихся процессов с непрерывным временем в случайной среде, у 
которых инфинитезимальные производяшие функции числа потомков имеют вид $f(s)=p_{0}+p_{1} s+p_{2} s^{2}$, был получен Н. А. Берестовой в [3].

Д ок а з а те л ь с т о. В ходе дальнейших рассуждений мы будем использовать свойства так называемого сопровождающего случайного блуждания $S_{k}, k \geqslant 0$, задаваемого при помоши соотношений

$$
S_{0}=0, \quad S_{k}=X_{1}+X_{2}+\cdots+X_{k}, \quad k \geqslant 1
$$

Заметим, что при любом $0 \leqslant k \leqslant n v$

$$
1-f_{0, n v}(0)=\mathbf{P}_{\mathscr{F}}\left\{Z_{n v} \geqslant 1\right\} \leqslant \mathbf{P}_{\mathscr{F}}\left\{Z_{k} \geqslant 1\right\} \leqslant \mathbf{E}_{\mathscr{F}} Z_{k}=e^{S_{k}}
$$

и, следовательно,

$$
\ln \left(1-f_{0, n v}(0)\right) \leqslant \min _{0 \leqslant k \leqslant n v} S_{k} .
$$

По условиям леммы случайное блуждание $S_{k}$ имеет нулевой снос и положительную конечную дисперсию $\sigma^{2}$. Отсюда и из принципа инвариантности для случайных блужданий вытекает (см., например, [4]), что для любого $x>0$

$$
\begin{aligned}
& \liminf _{n \rightarrow \infty} \mathbf{P}\left\{\frac{1}{\sigma \sqrt{n}} \ln \left(1-f_{0, n v}(0)\right)<x\right\} \\
& \geqslant \liminf _{n \rightarrow \infty} \mathbf{P}\left\{\frac{1}{\sigma \sqrt{n}} \min _{0 \leqslant u \leqslant v} S_{n u}<x\right\} \geqslant \mathbf{P}\left\{\inf _{0 \leqslant u \leqslant v} W(u)<x\right\} .
\end{aligned}
$$

Для получения оценки сверху напомним, что, согласно соотношениям (2.2) и (2.3) работы [26], при любом $m$

$$
\begin{aligned}
1-f_{0, m}(0) & \geqslant\left(e^{-S_{m}}+\sum_{k=0}^{m-1} 2 \eta_{k} e^{-S_{k}}\right)^{-1} \\
& \geqslant \exp \left\{\min _{0 \leqslant k \leqslant m} S_{k}\right\}\left(1+2 \sum_{k=0}^{m-1} \eta_{k}\right)^{-1} .
\end{aligned}
$$

Отсюда вытекает, что

$$
\ln \left(1-f_{0, n v}(0)\right) \geqslant \min _{0 \leqslant k \leqslant n v} S_{k}-\ln \left(1+2 \sum_{k=0}^{n v-1} \eta_{k}\right)
$$

Поскольку случайные величины $\eta_{k}, k=0,1,2, \ldots$, неотрицательны, независимы и одинаково распределены, то

$$
\mathbf{P}\left\{1+2 \sum_{k=0}^{n v-1} \eta_{k}>n^{2}\right\} \leqslant \frac{2}{n}\left(1+\mathbf{E} \eta_{1}\right) .
$$


Опираясь на эту оценку, нетрудно показать, что для любых $x<0$ и $\varepsilon \in(0,-x)$

$$
\begin{aligned}
& \mathbf{P}\left\{\frac{1}{\sigma \sqrt{n}} \ln \left(1-f_{0, n v}(0)\right)<x\right\} \\
& \quad \leqslant \mathbf{P}\left\{\frac{1}{\sigma \sqrt{n}} \min _{0 \leqslant u \leqslant v} S_{n u}<x+\varepsilon\right\}+O\left(n^{-1}\right) .
\end{aligned}
$$

Отсюда, устремляя сначала $n$ к бесконечности, а затем $\varepsilon$ к нулю, получаем

$$
\limsup _{n \rightarrow \infty} \mathbf{P}\left\{\frac{1}{\sigma \sqrt{n}} \ln \left(1-f_{0, n v}(0)\right)<x\right\} \leqslant \mathbf{P}\left\{\inf _{0 \leqslant u \leqslant v} W(u)<x\right\},
$$

что в сочетании с (15) доказывает лемму.

Следствие 1. В условиях леммы 2

$$
\left(\frac{1}{\sigma \sqrt{n}} \ln \left(1-f_{0, n v}(0)\right), v \in[0,1]\right) \stackrel{D}{\longrightarrow}\left(\inf _{0 \leqslant u \leqslant v} W(u), v \in[0,1]\right) .
$$

Д о к а 3 а т е л ь с т в о. Сходимость конечномерных распределений процесса $(\sigma \sqrt{n})^{-1} \ln \left(1-f_{0, n v}(0)\right)$ к соответствующим конечномерным распределениям процесса $\inf _{0 \leqslant u \leqslant v} W(u)$ легко установить, используя схему доказательства леммы 2 и неравенства (14) и (17). Этого достаточно для справедливости (21). Действительно, неравенства (14), (17) и (18) сводят задачу проверки плотности мер, порождаемых последовательностью процессов

$$
\alpha_{n}(t)=(\sigma \sqrt{n})^{-1} \ln \left(1-f_{0, n t}(0)\right), \quad t \in[0,1], n=1,2, \ldots,
$$

к проверке плотности мер процессов

$$
\beta_{n}(t)=(\sigma \sqrt{n})^{-1} \min _{0 \leqslant k \leqslant n t} S_{k}, \quad t \in[0,1], n=1,2, \ldots
$$

Последняя последовательность мер, очевидно, плотна.

Доказательство следствия закончено.

В последующих рассуждениях важное значение имеют свойства функции

$$
h(x)=\sum_{j=0}^{\infty} \mathbf{P}\left\{S_{\tau_{j}} \geqslant-x\right\}, \quad x \in \mathbf{R},
$$

где $0=\tau_{0}<\tau_{1}<\cdots$ - строгие нижние лестничные моменты случайного блуждания $\left\{S_{n}\right\}$, задаваемые соотношениями

$$
\tau_{j+1}:=\min \left\{n>\tau_{j}: S_{n}<S_{\tau_{j}}\right\}, \quad j \geqslant 0 .
$$


Пусть $M_{n}=\min \left(S_{0}, S_{1}, \ldots, S_{n}\right)$ и

$$
m_{n}(x)=\mathbf{P}\left\{M_{n} \geqslant-x\right\}, \quad x \geqslant 0 .
$$

Известно (см., например, теорему А работы [7]), что если $\mathbf{E} X_{1}=0$ и $0<\mathbf{E} X_{1}^{2}<\infty$, то найдутся константы $c_{1}$ и $c_{2}$ такие, что

$$
\begin{aligned}
& m_{n}(x) \sim c_{1} h(x) n^{-1 / 2}, \quad x \geqslant 0, n \rightarrow \infty \\
& m_{n}(x) \leqslant c_{2} h(x) n^{-1 / 2}, \quad x \geqslant 0, n \geqslant 1 .
\end{aligned}
$$

Заметим также, что из доказательства основной теоремы работы [26] вытекает, что величина $\beta$ в соотношении (2) имеет вид

$$
\beta=c_{1} \lim _{x \rightarrow \infty} \lim _{m \rightarrow \infty} \mathbf{E}\left[\left(1-f_{0, m}(0)\right) h\left(x+S_{m}\right) I\left(M_{m}>-x\right)\right],
$$

где константа $c_{1}$ та же, что и в $(22)$.

Нам понадобятся также следуюшие два утверждения, легко вытекающие из результатов работ [17] и [19].

Пусть

$$
Y_{n}(t)=\frac{1}{\sigma \sqrt{n}} S_{n t}, \quad \xi_{n}(t)=\frac{1}{\sigma \sqrt{n}} \min _{t n \leqslant k \leqslant n} S_{k}, \quad t \in[0,1] .
$$

Лемма 3. Если $\mathbf{E} X_{1}=0 u 0<\mathbf{E} X_{1}^{2}<\infty$, mо для любых $0<t_{1}<$ $t_{2}<\cdots<t_{r} \leqslant 1$, ^юбых $y_{1}, \ldots, y_{r} \in \mathbf{R}$ и любого $x \geqslant 0$

$$
\begin{aligned}
& \lim _{n \rightarrow \infty} \mathbf{P}\left\{Y_{n}\left(t_{1}\right)>y_{1}, \ldots, Y_{n}\left(t_{r}\right)>y_{r} \mid M_{n}>-x\right\} \\
& \quad=\mathbf{P}\left\{W^{+}\left(t_{1}\right)>y_{1}, \ldots, W^{+}\left(t_{r}\right)>y_{r}\right\} .
\end{aligned}
$$

Лемма 4. Eсли $\mathbf{E} X_{1}=0 u 0<\mathbf{E} X_{1}^{2}<\infty$, mо для любых $0<t_{1}<$ $t_{2}<\cdots<t_{r} \leqslant 1$, любых $y_{1}, \ldots, y_{r} \in \mathbf{R}$ и любого $x \geqslant 0$

$$
\begin{aligned}
& \lim _{n \rightarrow \infty} \mathbf{P}\left\{\xi_{n}\left(t_{1}\right)>y_{1}, \ldots, \xi_{n}\left(t_{r}\right)>y_{r} \mid M_{n}>-x\right\} \\
& \quad=\mathbf{P}\left\{\inf _{t_{1} \leqslant u \leqslant 1} W^{+}(u)>y_{1}, \ldots, \inf _{t_{r} \leqslant u \leqslant 1} W^{+}(u)>y_{r}\right\} .
\end{aligned}
$$

Теперь у нас все готово для доказательства еще одного важного утверждения.

Лемма 5. Если $\mathbf{E} X_{1}=0 u 0<\mathbf{E} X_{1}^{2}<\infty$, то для любых $0<t_{1}<$ $t_{2}<\cdots<t_{r} \leqslant 1$ и любых $y_{1}, \ldots, y_{r} \in \mathbf{R}$ справедливо соотношение

$$
\begin{gathered}
\lim _{n \rightarrow \infty} \mathbf{P}\left\{Y_{n}\left(t_{1}\right)>y_{1}, \ldots, Y_{n}\left(t_{r}\right)>y_{r} \mid Z_{n}>0\right\} \\
=\mathbf{P}\left\{W^{+}\left(t_{1}\right)>y_{1}, \ldots, W^{+}\left(t_{r}\right)>y_{r}\right\} .
\end{gathered}
$$


Д о к а з а т е л с т в о. При доказательстве этой леммы существенным образом использованы результаты работы [26]. Очевидно, что

$$
\begin{array}{r}
\mathbf{P}\left\{Y_{n}\left(t_{1}\right)>y_{1}, \ldots, Y_{n}\left(t_{r}\right)>y_{r} \mid Z_{n}>0\right\} \\
=\frac{1}{\mathbf{P}\left\{Z_{n}>0\right\}} \mathbf{E}\left[\left(1-f_{0, n}(0)\right) I(n ; \overline{\mathbf{t}}, \overline{\mathbf{y}})\right],
\end{array}
$$

где

$$
\begin{aligned}
I(n ; \overline{\mathbf{t}}, \overline{\mathbf{y}}) & =I\left(n ; t_{1}, \ldots, t_{r}, y_{1}, \ldots, y_{r}\right) \\
& =I\left(Y_{n}\left(t_{1}\right)>y_{1}, \ldots, Y_{n}\left(t_{r}\right)>y_{r}\right) .
\end{aligned}
$$

Зафиксируем натуральное число $m<n$, положительное число $x$ и $\gamma>1$ и представим второй сомножитель в правой части (28) в виде

$$
\begin{aligned}
\mathbf{E}\left[\left(1-f_{0, n}(0)\right) I(n ; \overline{\mathbf{t}}, \overline{\mathbf{y}})\right]= & G_{1}(n, x)+G_{2}(m, n, x, \gamma) \\
& +G_{3}(n, x, \gamma)+G_{4}(m, n, x, \gamma),
\end{aligned}
$$

где

$$
\begin{aligned}
G_{1}(n, x) & =\mathbf{E}\left[\left(1-f_{0, n}(0)\right) I(n ; \overline{\mathbf{t}}, \overline{\mathbf{y}}) I\left(M_{n} \leqslant-x\right)\right], \\
G_{2}(m, n, x, \gamma) & =\mathbf{E}\left[\left(f_{0, n}(0)-f_{0, m}(0)\right) I(n ; \overline{\mathbf{t}}, \overline{\mathbf{y}}) I\left(M_{n \gamma}>-x\right)\right], \\
G_{3}(n, x, \gamma) & =\mathbf{E}\left[\left(1-f_{0, n}(0)\right) I(n ; \overline{\mathbf{t}}, \overline{\mathbf{y}})\left(I\left(M_{n}>-x\right)-I\left(M_{n \gamma}>-x\right)\right)\right]
\end{aligned}
$$

и, наконец,

$$
G_{4}(m, n, x, \gamma)=\mathbf{E}\left[\left(1-f_{0, m}(0)\right) I(n ; \overline{\mathbf{t}}, \overline{\mathbf{y}}) I\left(M_{n \gamma}>-x\right)\right] .
$$

Очевидно, $G_{1}(n, x) \leqslant \mathbf{E}\left[\left(1-f_{0, n}(0)\right) I\left(M_{n} \leqslant-x\right)\right]$. Отсюда, используя неравенство (4.16) работы [26], получаем

$$
\limsup _{x \rightarrow \infty} \limsup _{n \rightarrow \infty} \sqrt{n} G_{1}(n, x)=0 .
$$

В силу леммы 4.2 из [26] для любого $\gamma>1$ и $x \geqslant 0$

$$
\limsup _{m \rightarrow \infty} \limsup _{n \rightarrow \infty} \sqrt{n} \mathbf{E}\left[\left(f_{0, n}(0)-f_{0, m}(0)\right) I\left(M_{n \gamma}>-x\right)\right]=0 .
$$

Отсюда и из неравенства

$$
G_{2}(m, n, x, \gamma) \leqslant \mathbf{E}\left[\left(f_{0, n}(0)-f_{0, m}(0)\right) I\left(M_{n \gamma}>-x\right)\right]
$$

вытекает, что

$$
\limsup _{m \rightarrow \infty} \limsup _{n \rightarrow \infty} \sqrt{n} G_{2}(m, n, x, \gamma)=0
$$


Вспоминая (22), нетрудно убедиться в том, что

$$
\begin{aligned}
G_{3}(n, x, \gamma) & \leqslant \mathbf{E}\left(I\left(M_{n}>-x\right)-I\left(M_{n \gamma}>-x\right)\right) \\
& \sim c_{1} h(x) n^{-1 / 2}\left(1-\gamma^{-1 / 2}\right)
\end{aligned}
$$

и, следовательно, для любого фиксированного $x \geqslant 0$

$$
\limsup _{\gamma \searrow 1} \limsup _{n \rightarrow \infty} \sqrt{n} G_{3}(n, x, \gamma)=0 .
$$

Займемся теперь исследованием асимптотического поведения величины $G_{4}(m, n, x, \gamma)$. Взяв условное математическое ожидание относительно $\sigma$-алгебры, порожденной случайными величинами $S_{0}, S_{1}, \ldots, S_{m}$ и $f_{0, m}(0)$, и вспомнив, что $\sigma \sqrt{n} Y_{n}(m / n)=S_{m}$, получаем

$$
\begin{aligned}
G_{4}(m, n, x, \gamma)=\mathbf{E} & {\left[\left(1-f_{0, m}(0)\right) I\left(M_{m}>-x\right)\right.} \\
& \left.\times P\left(n-m ; y_{1}-Y_{n}\left(\frac{m}{n}\right), \ldots, y_{r}-Y_{n}\left(\frac{m}{n}\right),-x-S_{m}\right)\right],
\end{aligned}
$$

где

$$
\begin{aligned}
& P\left(n-m ; y_{1}, \ldots, y_{r},-x\right) \\
& \quad=\mathbf{P}\left\{Y_{n}\left(t_{1}\right)-Y_{n}\left(\frac{m}{n}\right)>y_{1}, \ldots, Y_{n}\left(t_{r}\right)-Y_{n}\left(\frac{m}{n}\right)>y_{r} ;\right. \\
& \left.\quad \min _{m \leqslant k \leqslant n \gamma}\left(S_{k}-S_{m}\right)>-x\right\} \\
& =\mathbf{P}\left\{Y_{n}\left(t_{1}-\frac{m}{n}\right)>y_{1}, \ldots, Y_{n}\left(t_{1}-\frac{m}{n}\right)>y_{r} ; M_{n \gamma-m}>-x\right\} .
\end{aligned}
$$

Обращаясь к (22) и лемме 3 и полагая $S_{k}^{\prime}=S_{k+m}-S_{m}, k=0,1, \ldots$,

$$
M_{m}^{\prime}=\min _{1 \leqslant k \leqslant n} S_{k}^{\prime}, \quad Y_{n}^{\prime}(t)=\frac{1}{\sigma \sqrt{n}} S_{n t}^{\prime},
$$

заключаем, что при фиксированном $S_{m}$

$$
\begin{gathered}
\lim _{n \rightarrow \infty} \sqrt{n} P\left(n-m ; y_{1}-Y_{n}\left(\frac{m}{n}\right), \ldots, y_{r}-Y_{n}\left(\frac{m}{n}\right),-x-S_{m}\right) \\
=\lim _{n \rightarrow \infty} \mathbf{P}\left\{Y_{n}^{\prime}\left(t_{1}-\frac{m}{n}\right)>y_{1}, \ldots, Y_{n}^{\prime}\left(t_{r}-\frac{m}{n}\right)>y_{r} \mid\right. \\
\left.M_{n \gamma-m}^{\prime}>-x-S_{m}\right\} \lim _{n \rightarrow \infty} \sqrt{n} \mathbf{P}\left\{M_{n \gamma-m}^{\prime}>-x-S_{m}\right\} \\
=\frac{1}{\sqrt{\gamma}} \mathbf{P}\left\{\sqrt{\gamma} W^{+}\left(\frac{t_{1}}{\gamma}\right)>y_{1}, \ldots, \sqrt{\gamma} W^{+}\left(\frac{t_{r}}{\gamma}\right)>y_{r}\right\} c_{1} h\left(x+S_{m}\right) .
\end{gathered}
$$


Применяя теперь теорему об ограниченной сходимости и вспоминая (23), получаем

$$
\begin{aligned}
\lim _{n \rightarrow \infty} & \sqrt{n} G_{4}(m, n, x, \gamma) \\
= & (\sqrt{\gamma})^{-1} \mathbf{P}\left\{\sqrt{\gamma} W^{+}\left(\frac{t_{1}}{\gamma}\right)>y_{1}, \ldots, \sqrt{\gamma} W^{+}\left(\frac{t_{r}}{\gamma}\right)>y_{r}\right\} \\
& \times c_{1} \mathbf{E}\left[\left(1-f_{0, m}(0)\right) I\left(M_{m}>-x\right) h\left(x+S_{m}\right)\right] .
\end{aligned}
$$

Отсюда, обращаясь к определению величины $\beta$ (см. (24)), находим

$$
\begin{aligned}
& \lim _{x \rightarrow \infty} \lim _{m \rightarrow \infty} \lim _{\gamma \searrow 1} \lim _{n \rightarrow \infty} \sqrt{n} G_{4}(m, n, x, \gamma) \\
& \quad=\beta \mathbf{P}\left\{W^{+}\left(t_{1}\right)>y_{1}, W^{+}\left(t_{2}\right)>y_{2}, \ldots, W^{+}\left(t_{r}\right)>y_{r}\right\} .
\end{aligned}
$$

Объединяя $(31),(32),(34)$ и (38) и используя $(28)$ и $(24)$, приходим K (27).

Доказательство леммы закончено.

Положим

$$
\zeta_{n}(t)=\frac{1}{\sigma \sqrt{n}} \ln \left(Z_{n t} e^{-S_{n t}}\right), \quad t \in(0,1] .
$$

Только что доказанная лемма позволяет нам убедиться в справедливости следующего утверждения.

Лемма 6. Если выполнены условия теоремы 2, то для любого $\varepsilon>0$ июбого $t \in(0,1]$

$$
\lim _{n \rightarrow \infty} \mathbf{P}\left\{\left|\zeta_{n}(t)\right|>\varepsilon \mid Z_{n}>0\right\}=0 .
$$

Д о к а з а т е л ь с т в о. В силу неравенства Чебышева и (2)

$$
\begin{aligned}
& \mathbf{P}\left\{Z_{n t} e^{-S_{n t}}>e^{\varepsilon \sigma \sqrt{n}} \mid Z_{n}>0\right\} \leqslant \frac{1}{\mathbf{P}\left\{Z_{n}>0\right\}} \mathbf{P}\left\{Z_{n t} e^{-S_{n t}}>e^{\varepsilon \sigma \sqrt{n}}\right\} \\
& \quad \leqslant \frac{e^{-\varepsilon \sigma \sqrt{n}}}{\mathbf{P}\left\{Z_{n}>0\right\}} \mathbf{E} Z_{n t} e^{-S_{n t}} \leqslant c \sqrt{n} e^{-\varepsilon \sigma \sqrt{n}} \longrightarrow 0
\end{aligned}
$$

при $n \rightarrow \infty$. Таким образом,

$$
\lim _{n \rightarrow \infty} \mathbf{P}\left\{\zeta_{n}(t)>\varepsilon \mid Z_{n}>0\right\}=0 .
$$

Заметим теперь, что в соответствии с теоремой 2 и леммой 5

$$
\begin{aligned}
& \lim _{n \rightarrow \infty} \mathbf{P}\left\{\frac{1}{\sigma \sqrt{n}} \ln Z_{n t}>y \mid Z_{n}>0\right\} \\
& =\lim _{n \rightarrow \infty} \mathbf{P}\left\{\frac{1}{\sigma \sqrt{n}} \ln \left(Z_{n t} e^{-S_{n t}}\right)+\frac{1}{\sigma \sqrt{n}} S_{n t}>y \mid Z_{n}>0\right\} \\
& =\lim _{n \rightarrow \infty} \mathbf{P}\left\{\zeta_{n}(t)+Y_{n}(t)>y \mid Z_{n}>0\right\} \\
& =\lim _{n \rightarrow \infty} \mathbf{P}\left\{Y_{n}(t)>y \mid Z_{n}>0\right\}=\mathbf{P}\left\{W_{1}^{+}(t)>y\right\} .
\end{aligned}
$$


Докажем, что из (41) и (42) следует, что для любого $\varepsilon>0$

$$
\lim _{n \rightarrow \infty} \mathbf{P}\left\{\zeta_{n}(t)<-\varepsilon \mid Z_{n}>0\right\}=0 .
$$

Для фиксированных $\gamma>0$ и натурального $N$ имеем

$$
\begin{aligned}
& \mathbf{P}\left\{\zeta_{n}(t)<-\varepsilon \mid Z_{n}>0\right\}=\mathbf{P}\left\{Y_{n}(t)<\gamma, \zeta_{n}(t)<-\varepsilon \mid Z_{n}>0\right\} \\
& \quad+\sum_{j=0}^{N-1} \mathbf{P}\left\{Y_{n}(t) \in[\gamma+j \varepsilon, \gamma+(j+1) \varepsilon), \zeta_{n}(t)<-\varepsilon \mid Z_{n}>0\right\} \\
& \quad+\mathbf{P}\left\{Y_{n}(t) \geqslant \gamma+N \varepsilon, \zeta_{n}(t)<-\varepsilon \mid Z_{n}>0\right\} .
\end{aligned}
$$

Оценим каждое слагаемое в правой части (44). В силу (42) для любого $\delta>0$ найдутся достаточно малое $\gamma>0$ и достаточно большое натуральное $N$ такие, что

$$
\begin{array}{r}
\mathbf{P}\left(\left\{Y_{n}(t)<\gamma\right\} \cup\left\{Y_{n}(t) \geqslant \gamma+N \varepsilon\right\}, \zeta_{n}(t)<-\varepsilon \mid Z_{n}>0\right) \\
\leqslant \mathbf{P}\left(\left\{Y_{n}(t)<\gamma\right\} \cup\left\{Y_{n}(t) \geqslant \gamma+N \varepsilon\right\} \mid Z_{n}>0\right)<\delta .
\end{array}
$$

В дальнейшем будем считать, что $\gamma>0$ и $N$ выбраны именно таким образом. Для оценки оставшихся слагаемых заметим, что для любого $y>\varepsilon$ и любого $\varepsilon_{1}>0$

$$
\begin{aligned}
& \mathbf{P}\left\{\zeta_{n}(t)+Y_{n}(t)>y \mid Z_{n}>0\right\} \\
&= \mathbf{P}\left\{\zeta_{n}(t)+Y_{n}(t)>y, \zeta_{n}(t)<-\varepsilon \mid Z_{n}>0\right\} \\
&+\mathbf{P}\left\{\zeta_{n}(t)+Y_{n}(t)>y, \zeta_{n}(t) \in\left[-\varepsilon, \varepsilon_{1}\right) \mid Z_{n}>0\right\} \\
&+\mathbf{P}\left\{\zeta_{n}(t)+Y_{n}(t)>y, \zeta_{n}(t) \geqslant \varepsilon_{1} \mid Z_{n}>0\right\} \\
& \leqslant \mathbf{P}\left\{Y_{n}(t)>y+\varepsilon, \zeta_{n}(t)<-\varepsilon \mid Z_{n}>0\right\} \\
&+\mathbf{P}\left\{Y_{n}(t)>y-\varepsilon_{1}, \zeta_{n}(t) \geqslant-\varepsilon \mid Z_{n}>0\right\} \\
&+\mathbf{P}\left\{\zeta_{n}(t) \geqslant \varepsilon_{1} \mid Z_{n}>0\right\} .
\end{aligned}
$$

Следовательно,

$$
\begin{aligned}
& \mathbf{P}\left\{\zeta_{n}(t)+Y_{n}(t)>y \mid Z_{n}>0\right\} \leqslant \mathbf{P}\left\{Y_{n}(t)>y-\varepsilon_{1} \mid Z_{n}>0\right\} \\
& \quad-\mathbf{P}\left\{Y_{n}(t) \in\left(y-\varepsilon_{1}, y+\varepsilon\right], \zeta_{n}(t)<-\varepsilon \mid Z_{n}>0\right\} \\
& \quad+\mathbf{P}\left\{\zeta_{n}(t) \geqslant \varepsilon_{1} \mid Z_{n}>0\right\} .
\end{aligned}
$$

Пусть возрастаюшая последовательность натуральных чисел $n_{k}, k=$ $1,2, \ldots$, такова, что сушествует предел

$$
c=\lim _{n_{k} \rightarrow \infty} \mathbf{P}\left\{Y_{n_{k}}(t) \in[y, y+\varepsilon], \zeta_{n_{k}}(t)<-\varepsilon \mid Z_{n_{k}}>0\right\} .
$$


Покажем, что $c=0$. Действительно, переходя к пределу при $n_{k} \rightarrow \infty$ в обеих частях неравенства (47) и учитывая (43) и (44), получим

$$
\begin{aligned}
\mathbf{P}\left\{W_{1}^{+}(t)>y\right\}= & \lim _{n_{k} \rightarrow \infty} \mathbf{P}\left\{\zeta_{n_{k}}(t)+Y_{n_{k}}(t)>y \mid Z_{n_{k}}>0\right\} \\
\leqslant & \lim _{n_{k} \rightarrow \infty} \mathbf{P}\left\{Y_{n_{k}}(t)>y-\varepsilon_{1} \mid Z_{n_{k}}>0\right\} \\
& -c+\lim _{n_{k} \rightarrow \infty} \mathbf{P}\left\{\zeta_{n_{k}}(t) \geqslant \varepsilon_{1} \mid Z_{n_{k}}>0\right\} \\
= & \mathbf{P}\left\{W_{1}^{+}(t)>y-\varepsilon_{1}\right\}-c .
\end{aligned}
$$

Устремляя $\varepsilon_{1}$ к нулю, заключаем, что

$$
\mathbf{P}\left\{W_{1}^{+}(t)>y\right\} \leqslant \mathbf{P}\left\{W_{1}^{+}(t)>y\right\}-c .
$$

Отсюда, в силу неотрицательности $c$ следует, что $c=0$. Последнее, в силу произвольности подпоследовательности $n_{k}$, для которой существует предел в (48), влечет

$$
\lim _{n \rightarrow \infty} \mathbf{P}\left\{Y_{n}(t) \in[y, y+\varepsilon), \zeta_{n}(t)<-\varepsilon \mid Z_{n}>0\right\}=0
$$

для любого $y>0$. В частности, для любого $j=1,2, \ldots, N-1$

$$
\lim _{n \rightarrow \infty} \mathbf{P}\left\{Y_{n}(t) \in[\gamma+j \varepsilon, \gamma+(j+1) \varepsilon), \zeta_{n}(t)<-\varepsilon \mid Z_{n}>0\right\}=0 .
$$

Подставляя (45) и (51) в (44), приходим к (43).

Лемма доказана.

Лемма 7. Если $\mathbf{E} X_{1}=0 u 0<\mathbf{E} X_{1}^{2}<\infty$, mо для любых $0<t_{1}<$ $t_{2}<\cdots<t_{r} \leqslant 1$ и ююбих $y_{1}, \ldots, y_{r} \in \mathbf{R}$ справедливо соотношение

$$
\begin{aligned}
& \lim _{n \rightarrow \infty} \mathbf{P}\left\{\xi_{n}\left(t_{1}\right)>y_{1}, \ldots, \xi_{n}\left(t_{r}\right)>y_{r} \mid Z_{n}>0\right\} \\
& \quad=\mathbf{P}\left\{\inf _{t_{1} \leqslant u \leqslant 1} W^{+}(u)>y_{1}, \ldots, \inf _{t_{r} \leqslant u \leqslant 1} W^{+}(u)>y_{r}\right\} .
\end{aligned}
$$

Аргументы, необходимые для доказательства этой леммы, практически не отличаются от рассуждений, использовавшихся при доказательстве леммы 5, и мы их опускаем. Отметим лишь, что при доказательстве необходимо вместо леммы 3, на которой базировалось доказательство леммы 5 , применить лемму 4.

2. Доказательство основного результата. Легко понять, что для доказательства основного результата работы - теоремы 3 достаточно установить справедливость ее утверждения для процесса $(\sigma \sqrt{n})^{-1} \ln \left(e+Z_{n t, n}\right)$ вместо процесса $(\sigma \sqrt{n})^{-1} \ln Z_{n t, n}$. Именно так мы и поступим. Займемся сходимостью одномерных распределений. 
Теорема 4. В условиях теоремь 3 для любого $t \in[0,1] u$ любого $x>0$

$$
\lim _{n \rightarrow \infty} \mathbf{P}\left\{\frac{1}{\sigma \sqrt{n}} \ln \left(e+Z_{n t, n}\right)>x \mid Z_{n}>0\right\}=\mathbf{P}\left\{\inf _{t \leqslant u \leqslant 1} W^{+}(u)>x\right\} .
$$

Д о к а з а т е л ь с т в о. Как следует из леммы 1 , неравенств

$$
\begin{aligned}
& \mathbf{P}\left(A_{n, t}(x) \overline{B_{n, t}(N)} \overline{C_{n, t}(\delta)}\right) \leqslant \mathbf{P}\left(A_{n, t}(x)\right) \leqslant \mathbf{P}\left(A_{n, t}(x) \overline{B_{n, t}(N)} \overline{C_{n, t}(\delta)}\right) \\
& \quad+\mathbf{P}\left(A_{n, t}(x) B_{n, t}(N)\right)+\mathbf{P}\left(A_{n, t}(x) C_{n, t}(\delta)\right)
\end{aligned}
$$

и включения $A_{n, t}(x) \subset\left\{Z_{n}>0\right\}$, справедливого для фиксированных $x>0$ и $t \in[0,1]$ и всех достаточно больших $n=n(x)$, для доказательства теоремы достаточно показать, что

$$
\lim _{\delta \rightarrow 0} \lim _{N \rightarrow \infty} \lim _{n \rightarrow \infty} \frac{\mathbf{P}\left(A_{n, t}(x) \overline{B_{n, t}(N)} \overline{C_{n, t}(\delta)}\right)}{\mathbf{P}\left\{Z_{n}>0\right\}}=\mathbf{P}\left\{\inf _{t \leqslant u \leqslant 1} W^{+}(u)>x\right\} .
$$

Используя равенство

$$
Z_{n t, n}=Z_{n t}\left(1-f_{n t, n}(0)\right)+\sum_{k=1}^{Z_{n t}}\left(I\left(Z_{n t, n}^{(k)}>0\right)-\left(1-f_{n t, n}(0)\right)\right)
$$

неравенство

$$
\left|\sum_{k=1}^{Z_{n t}}\left(I\left(Z_{n t, n}^{(k)}>0\right)-\left(1-f_{n t, n}(0)\right)\right)\right| \leqslant \sqrt{N Z_{n t}\left(1-f_{n t, n}(0)\right) f_{n t, n}(0)},
$$

справедливое на множестве $\overline{B_{n, t}(N)}$, и вспоминая (7), (8) и тот факт, что $Z_{n t}\left(1-f_{n t, n}(0)\right) \geqslant e^{n^{1 / 4-\delta}}$ на множестве $\overline{C_{n, t}(\delta)} \cap\left\{Z_{n t}>0\right\}$, заключаем, что для любого $\varepsilon>0$ найдется $n_{0}=n_{0}(\varepsilon)$ такое, что при $n \geqslant n_{0}$

$$
\begin{aligned}
\mathbf{P} & \left(A_{n, t}(x) \overline{B_{n, t}(N)} \overline{C_{n, t}(\delta)}\right) \\
\leqslant & \mathbf{P}\left\{\ln \left(e+Z_{n t}\left(1-f_{n t, n}(0)\right)(1+\varepsilon)\right)>x \sqrt{n} ; \overline{C_{n, t}(\delta)} ; Z_{n}>0\right\} \\
& +\alpha_{1}(n, N, \delta) \\
\leqslant & \mathbf{P}\left\{\ln Z_{n t}+\ln \left(1-f_{n t, n}(0)\right)+\ln (1+2 \varepsilon)>x \sqrt{n} ; Z_{n}>0\right\} \\
& +\alpha_{1}(n, N, \delta),
\end{aligned}
$$

где $\lim \sup _{\delta \rightarrow 0} \lim \sup _{N \rightarrow \infty} \lim \sup _{n \rightarrow \infty} \sqrt{n} \alpha_{1}(n, N, \delta)=0$. Отсюда, из (2), леммы 6 , неравенств (20) и (17) вытекает, что

$$
\begin{aligned}
& \limsup _{\delta \rightarrow 0} \limsup _{N \rightarrow \infty} \limsup _{n \rightarrow \infty} \frac{\mathbf{P}\left(A_{n, t}(x) \overline{B_{n, t}(N)} \overline{C_{n, t}(\delta)}\right)}{\mathbf{P}\left\{Z_{n}>0\right\}} \\
& \quad \leqslant \lim _{n \rightarrow \infty} \mathbf{P}\left\{\frac{1}{\sigma \sqrt{n}} \ln Z_{n t}+\frac{1}{\sigma \sqrt{n}} \ln \left(1-f_{n t, n}(0)\right)>x \mid Z_{n}>0\right\}
\end{aligned}
$$




$$
\begin{aligned}
& =\lim _{n \rightarrow \infty} \mathbf{P}\left\{\frac{1}{\sigma \sqrt{n}} S_{n t}+\frac{1}{\sigma \sqrt{n}} \min _{n t \leqslant k \leqslant n}\left(S_{k}-S_{n t}\right)>x \mid Z_{n}>0\right\} \\
& =\lim _{n \rightarrow \infty} \mathbf{P}\left\{\frac{1}{\sigma \sqrt{n}} \min _{n t \leqslant k \leqslant n} S_{k}>x \mid Z_{n}>0\right\} \\
& =\mathbf{P}\left\{\inf _{t \leqslant u \leqslant 1} W^{+}(u) \geqslant x\right\} .
\end{aligned}
$$

По тем же соображениям,

$$
\begin{aligned}
& \mathbf{P}\left(A_{n, t}(x) \overline{B_{n, t}(N)} \overline{C_{n, t}(\delta)}\right) \\
& \geqslant \mathbf{P}\left\{\ln \left(e+Z_{n t}\left(1-f_{n t, n}(0)\right)(1-\varepsilon)\right)>x \sqrt{n}, \overline{B_{n, t}(N)} \overline{C_{n, t}(\delta)} ; Z_{n}>0\right\} \\
& \geqslant \mathbf{P}\left\{\ln Z_{n t}+\ln \left(1-f_{n t, n}(0)\right)+\ln (1-2 \varepsilon)>x \sqrt{n} ; Z_{n}>0\right\} \\
& \quad+\alpha_{2}(n, N, \delta),
\end{aligned}
$$

где

$$
\liminf _{\delta \rightarrow 0} \liminf _{N \rightarrow \infty} \liminf _{n \rightarrow \infty} \sqrt{n} \alpha_{2}(n, N, \delta)=0 .
$$

Отсюда вытекает, что

$$
\begin{aligned}
& \liminf _{\delta \rightarrow 0} \liminf _{N \rightarrow \infty} \liminf _{n \rightarrow \infty} \frac{\mathbf{P}\left(A_{n, t}(x) \overline{B_{n, t}(N)} \overline{C_{n, t}(\delta)}\right)}{\mathbf{P}\left\{Z_{n}>0\right\}} \\
& \geqslant \mathbf{P}\left\{\inf _{t \leqslant u \leqslant 1} W^{+}(u) \geqslant x\right\} .
\end{aligned}
$$

Объединяя (57) и (58) получаем желаемую сходимость одномерных распределений.

Теорема доказана.

Практически дословное повторение рассуждений, использовавшихся при доказательстве предыдушей теоремы, позволяет убедиться в справедливости следующего утверждения.

Теорема 5. В условиях теоремь 3 для любых $0<t_{1}<\cdots<t_{r} \leqslant 1$ $u y_{1}, \ldots, y_{r} \in \mathbf{R}$

$$
\begin{gathered}
\lim _{n \rightarrow \infty} \mathbf{P}\left\{\frac{1}{\sigma \sqrt{n}} \ln \left(e+Z_{n t_{1}, n}\right)>y_{1}, \ldots, \frac{1}{\sigma \sqrt{n}} \ln \left(e+Z_{n t_{r}, n}\right)>y_{r} \mid Z_{n}>0\right\} \\
=\mathbf{P}\left\{\inf _{t_{1} \leqslant u \leqslant 1} W^{+}(u)>y_{1}, \ldots, \inf _{t_{r} \leqslant u \leqslant 1} W^{+}(u)>y_{r}\right\} .
\end{gathered}
$$

Д ок а з а те ль с т в о т е оремы 3 . Для монотонной функции $h \in D[0,1]$ и $\delta>0$ положим

$$
w^{\prime}(h, \delta)=\inf _{\left\{t_{i}\right\}} \max _{0<i \leqslant r}\left|h\left(t_{i}-\right)-h\left(t_{i-1}\right)\right|,
$$

где нижняя грань берется по всем конечным множествам $\left\{t_{i}\right\}$ точек, удовлетворяющих усповиям $0=t_{0}<t_{1}<\cdots<t_{r}=1, t_{i}-t_{i-1}>\delta$, 
$i=1,2, \ldots, r$. Согласно теореме 15.3 монографии [4], для доказательства плотности семейства мер, порождаемых монотонными по $t$ процессами

$$
\gamma_{n}(t)=\frac{1}{\sigma \sqrt{n}} \ln Z_{n t, n}, \quad n=1,2, \ldots,
$$

при условии $\left\{Z_{n}>0\right\}$, необходимо показать, что

i) для любого положительного $\eta$ существует $a=a(\eta)$ такое, что

$$
\mathbf{P}\left\{\sup _{t \in[0,1]}\left|\gamma_{n}(t)\right|>a \mid Z_{n}>0\right\} \leqslant \eta, \quad n \geqslant 1,
$$

ii) для любых положительных $\varepsilon$ и $\varepsilon_{1}$ существуют $\delta=\delta\left(\varepsilon, \varepsilon_{1}\right) \in(0,1]$ и целое число $n_{0}=n_{0}\left(\varepsilon, \varepsilon_{1}, \delta\right)$ такие, что

$$
\mathbf{P}\left\{w^{\prime}\left(\gamma_{n}, \delta\right) \geqslant \varepsilon \mid Z_{n}>0\right\} \leqslant \varepsilon_{1}, \quad n \geqslant n_{0} .
$$

Поскольку $0 \leqslant \gamma_{n}(t) \leqslant \gamma_{n}(1)=(\sigma \sqrt{n})^{-1} \ln Z_{n}$, то справедливость і) вытекает из предыдущей теоремы.

Доказательство того, что выполнено іi), требует бо́льших усилий. Будем искать нужное нам число $\delta$ среди элементов последовательности $\left\{r^{-1}\right\}, r=1,2, \ldots$ Ясно, что для любого $\delta=r^{-1}$

$\mathbf{P}\left\{w^{\prime}\left(\gamma_{n}, r^{-1}\right) \geqslant \varepsilon \mid Z_{n}>0\right\} \leqslant \mathbf{P}\left\{\max _{0<i \leqslant r}(\gamma(i \delta-)-\gamma((i-1) \delta)) \geqslant \varepsilon \mid Z_{n}>0\right\}$.

Отсюда, устремляя $n$ к бесконечности и вспоминая теорему 5 , получаем, что

$$
\begin{aligned}
& \limsup _{n \rightarrow \infty} \mathbf{P}\left\{w^{\prime}\left(\gamma_{n}, r^{-1}\right) \geqslant \varepsilon \mid Z_{n}>0\right\} \\
& \quad \leqslant \mathbf{P}\left\{\max _{0<i \leqslant r}\left(\inf _{i \delta \leqslant u \leqslant 1} W^{+}(u)-\inf _{(i-1) \delta \leqslant u \leqslant 1} W^{+}(u)\right) \geqslant \varepsilon\right) \\
& \quad=\mathbf{P}\left\{\max _{0<i \leqslant r}\left(\inf _{i \delta \leqslant \leqslant \leqslant 1} W^{+}(u)-\inf _{(i-1) \delta \leqslant u \leqslant i \delta} W^{+}(u)\right) \geqslant \varepsilon\right\} \\
& \leqslant \mathbf{P}\left\{\max _{0<i \leqslant r}\left(W^{+}(i \delta)-\inf _{(i-1) \delta \leqslant u \leqslant i \delta} W^{+}(u)\right) \geqslant \varepsilon\right\} \\
& \leqslant \sum_{i=1}^{r} \mathbf{P}\left\{W^{+}(i \delta)-\inf _{(i-1) \delta \leqslant u \leqslant i \delta} W^{+}(u) \geqslant \varepsilon\right\} \\
&=\sum_{i=1}^{r} \mathbf{P}\left\{\sup _{(i-1) \delta \leqslant u \leqslant i \delta}\left(W^{+}(i \delta)-W^{+}(u)\right) \geqslant \varepsilon\right\} .
\end{aligned}
$$

Заметим теперь, что, согласно лемме 3 работы [2],

$$
\lim _{\delta \downarrow 0} \frac{1}{\delta} \mathbf{P}\left\{\sup _{(r-1) \delta \leqslant u \leqslant 1}\left(W^{+}(1)-W^{+}(u)\right) \geqslant \varepsilon\right\}=0,
$$

и, кроме того,

$$
\lim _{\delta \downarrow 0} \frac{1}{\delta} \mathbf{P}\left\{\sup _{0 \leqslant u \leqslant \delta}\left(W^{+}(\delta)-W^{+}(u)\right) \geqslant \varepsilon\right\}=0 .
$$


Пусть, как и прежде, $W(t)$ стандартное броуновское движение на $[0,1]$, $W(0)=0$. Используя рассуждения, применявшиеся при доказательстве уже упоминавшейся леммы 3 работы [2], нетрудно показать, что при $1 \leqslant i \leqslant r-1$

$$
\begin{aligned}
& \mathbf{P}\left\{\sup _{(i-1) \delta \leqslant u \leqslant i \delta}\left(W^{+}(i \delta)-W^{+}(u)\right) \geqslant \varepsilon\right\} \\
& \quad \leqslant \frac{i}{i-1} \mathbf{P}\left\{\sup _{(i-1) \delta \leqslant u \leqslant i \delta}|W(i \delta)-W(u)| \geqslant \varepsilon\right\} \\
& \quad \leqslant 4 \mathbf{P}\left\{\sup _{(i-1) \delta \leqslant u \leqslant i \delta}|W(u)-W((i-1) \delta)| \geqslant \frac{\varepsilon}{2}\right\} \\
& \quad=4 \mathbf{P}\left\{\sup _{0 \leqslant u \leqslant \delta}|W(u)| \geqslant \frac{\varepsilon}{2}\right\}=4 \mathbf{P}\left\{\sup _{0 \leqslant u \leqslant 1}|W(u)| \geqslant \frac{\varepsilon}{2 \sqrt{\delta}}\right\},
\end{aligned}
$$

причем (см., например, $[4, \S 10])$

$$
\lim _{\delta \downarrow} \frac{1}{\delta} \mathbf{P}\left\{\sup _{0 \leqslant u \leqslant 1}|W(u)| \geqslant \frac{\varepsilon}{2 \sqrt{\delta}}\right\}=0 .
$$

Собирая соотношения (64)-(67), подставляя их в (63) и вспоминая, что $\delta=r^{-1}$, получаем

$$
\begin{aligned}
& \underset{r \rightarrow \infty}{\limsup \limsup } \sup _{n \rightarrow \infty} \mathbf{P}\left\{w^{\prime}\left(\gamma_{n}, r^{-1}\right) \geqslant \varepsilon \mid Z_{n}>0\right\} \\
& \quad \leqslant \operatorname{lim\operatorname {sup}}\left(4\left(\delta^{-1}-2\right) \mathbf{P}\left\{\sup _{\delta \downarrow 0}|W(u)| \geqslant \frac{\varepsilon}{2 \sqrt{\delta}}\right\}\right)=0,
\end{aligned}
$$

что, очевидно, влечет (62).

Таким образом, условия і) и іi) выполнены, а значит, плотность мер, порождаемых условными процессами $\gamma_{n}(t)$, доказана.

Отсюда и из теоремы 5 с учетом того, что на интересующих нас множествах процессы $(\sigma \sqrt{n})^{-1} \ln \left(e+Z_{[n t], n}\right)$ и $(\sigma \sqrt{n})^{-1} \ln Z_{[n t], n}$ асимптотически эквивалентны, следует (5).

Теорема 3 доказана.

В заключение автор хотел бы выразить благодарность В. А. Афанасьеву за ряд полезных замечаний, способствовавших улучшению изложения представленных здесь результатов.

\section{СПИСОК ЛИТЕРАТУРЫ}

1. Афанасьев В.И. Предельная теорема для критического ветвящегося процесса в случайной среде. - Дискретн. матем., 1993, т. 5, № 1, с. 45-58.

2. АФанасьев В.И. О максимуме критического ветвящегося процесса в случайной среде. - Дискретн. матем., 1999, т. 11, № 2, с. 86-102. 
3. Берестова Н. А. О больших уклонениях для ветвяшихся процессов на конечном интервале времени. - Докл. АН СССР, 1981, т. 261, №6, с. 1289-1292.

4. Биллингсли П. Сходимость вероятностных мер. М.: Наука, 1977, 352 с.

5. Ватутин B. A. О расстоянии до ближайшего общего предка в ветвящихся процессах Беллмана-Харриса. - Матем. заметки, 1979, т. 25, № 5, с. 733-741.

6. Зубков А.М. Предельные распределения расстояния до ближайшего общего предка. - Теория вероятн. и ее примен., 1975, т. 20, № 3, с. 614-623.

7. Козлов М.В. Об асимптотике вероятности невырождения критических ветвящихся процессов в случайной среде. - Теория вероятн. и ее примен., 1976, т. 21, № 4, c. $813-825$.

8. Козлов М.В. Условная функциональная предельная теорема для критического ветвящегося процесса в случайной среде. - Докл. РАН, 1995, т. 344, № 1, с. 12 15.

9. Сагитов С.М. Общие предки в критических ветвящихся процессах БеллманаХарриса с несколькими типами частиц. - Изв. АН КазССР, сер. физ.-матем. наук, 1982 , т. 3 , с. $66-69$.

10. Сагитов С. М. Новая предельная теорема для редуцированных критических ветвящихся процессов. -- Изв. АН КазССР, сер. физ.-матем. наук, 1989, т. 3, с. 3336.

11. Сагитов С.M. Редуцированный критический ветвящийся процесс БеллманаХарриса с несколькими типами частиц. - Теория вероятн. и ее примен., 1985, т. 30, № 4, c. 737-749.

12. Сагитов С. М. Предельное поведение редуцированных критических ветвящихся процессов. - Докл. АН СССР, 1988, т. 303, № 3, с. 47-49.

13. Сагитов С.М. Три предельные теоремы для редуцированных критических ветвящихся процессов. - Успехи мат. наук, 1995, т. 50, в. 5, с. 183-202.

14. Якымив А.Л. Редуцированные ветвящиеся процессы. - Теория вероятн. и ее примен., 1980 , т. 25 , в. 3, с. 593-596.

15. Якьмив А.Л. Асимптотические свойства докритических и надкритических редуцированных ветвящихся процессов. - Теория вероятн. и ее примен., 1985, т. 30 , B. 1 , c. $183-188$.

16. Athreya K. B., Ney P. E. Branching Processes. Berlin: Springer-Verlag, 1972, 287 p.

17. Bolthausen $E$. On a functional central limit theorem for random walks conditioned to stay positive. - Ann. Probab., 1976, т. 4, № 3, p. 480-485.

18. Borovkov K. A., Vatutin V.A. Reduced critical branching processes in random environment. - Stochastic Process. Appl., 1997, т. 71, № 2, p. 225-240.

19. Durrett R. T., Iglehart D. L., Miller D. R. Weak convergence to Brownian meander and Brownian excursion. - Ann. Probab., 1977, т. 5, № 1, p. 117-129.

20. D'Souza J. C., Hambly B. M. On the survival probability of a branching process in a random environment. - Adv. Appl. Probab., 1997, т. 29, № 1, p. 38-55.

21. Fleischmann K., Vatutin V. A. Reduced subcritical Galton-Watson processes in random environment. - Adv. Appl. Probab., 1999, т. 31, № 1, p. 88-111.

22. Fleischmann K., Prehn U. Ein Grenzwertsatz für subkritische Verzweigungsprozesse mit eindlich vielen Typen von Teilchen. - Math. Nachr., 1977, т. 79, p. 357-362.

23. Fleischmann K., Siegmund-Schultze R. The structure of reduced critical GaltonWatson processes. -- Math. Nachr., 1977, т. 79, p. 233-241.

24. Guivarc'h $Y$., Liu $Q$. Propriétés asymptotiques des processus de branchement en environnement aléatoire. - C. R. Acad. Sci. Paris Sér. I Math., 2001, v. 332, № 4, p. 339-344.

25. Liu $Q$. On the survival probability of a branching process in a random environment. Ann. Inst. H. Poincaré Probab. Statist., 1996, v. 32, № 1, p. 1-10.

26. Geiger J., Kersting G. The survival probability of a critical branching process in random environment. - Теория вероятн. и ее примен., 2000, v. 45, № 3, р. 607 615. 